\title{
On The Effect of Giant Planets on the Scattering of Parent Bodies of Iron Meteorite from the Terrestrial Planet Region into the Asteroid Belt: A Concept Study
}

\author{
Nader Haghighipour \\ Institute for Astronomy and NASA Astrobiology Institute, University of Hawaii-Manoa, \\ Honolulu, HI 96822, USA \\ nader@ifa.hawaii.edu \\ and \\ Edward R. D. Scott \\ Hawaii Institute for Geophysics and Planetology, University of Hawaii-Manoa, Honolulu, \\ HI 96822
}

\begin{abstract}
In their model for the origin of the parent bodies of iron meteorites, Bottke et al proposed differentiated planetesimals that were formed in the region of 1 $2 \mathrm{AU}$ during the first 1.5 Myr, as the parent bodies, and suggested that these objects and their fragments were scattered into the asteroid belt as a result of interactions with planetary embryos. Although viable, this model does not include the effect of a giant planet that might have existed or been growing in the outer regions. We present the results of a concept study where we have examined the effect of a planetary body in the orbit of Jupiter on the early scattering of planetesimals from terrestrial region into the asteroid belt. We integrated the orbits of a large battery of planetesimals in a disk of planetary embryos, and studied their evolutions for different values of the mass of the planet. Results indicate that when the mass of the planet is smaller than 10 Earth-masses, its effects on the interactions among planetesimals and planetary embryos is negligible. However, when the planet mass is between 10 and 50 Earthmasses, simulations point to a transitional regime with $\sim 50$ Earth-mass being the value for which the perturbing effect of the planet can no longer be ignored. Simulations also show that further increase of the mass of the planet strongly reduces the efficiency of the scattering of planetesimals from the terrestrial planet region into the asteroid belt. We present the results of our simulations and discuss their possible implications for the time of giant planet formation.
\end{abstract}


Subject headings: Asteroids: general, Asteroids: dynamics, Planets: dynamics, Meteorites, Methods: numerical

\section{Introduction}

It has been suggested by Bottke et al. (2006) that the extraordinary number of different parent bodies of iron meteorites, and the dearth of asteroids and meteorites derived from the silicate mantles and crusts of these objects, could be better understood if these bodies accreted not in the asteroid belt, but in the terrestrial planet region (1-2 AU). If the parent bodies of iron meteorites had formed and differentiated igneously in the main asteroid belt, we should have been able to find many metallic asteroids composed of debris from cores, as well as numerous olivine-rich asteroids and meteorites composed of mantle

material. Burbine et al. (1996) attributed the lack of olivine-rich and metal-rich asteroids to numerous impacts that pulverized the differentiated asteroids. However, this explanation appears inconsistent with the survival of Vesta's basaltic crust, and the existence of some unrelated V-type asteroids (Moskovitz et al. 2008) and basaltic meteorites that are not from Vesta.

A major argument of Bottke et al. (2006) for suggesting that the igneously differentiated asteroids formed at 1-2 AU is that parent bodies of iron meteorites accreted in the first 1.5 Myr (Kleine et al. 2005; Markowski et al. 2006a,b) when ${ }^{26} \mathrm{Al}$, which has a half-life of $0.73 \mathrm{Myr}$, was still capable of melting bodies larger than $20 \mathrm{~km}$ in radius (Hevey \& Sanders 2006). Accretion rates would have been much faster at 1-2 AU than in the asteroid belt due to the higher density of solids and the shorter orbital periods. Later accretion of planetesimals in the asteroid belt at 2-4 Myr, when ${ }^{26} \mathrm{Al}$ had largely decayed, accounts for the preponderance of unmelted asteroids in the belt and the relatively young ages of chondrules (e.g., Scott 2008).

Bottke et al. (2006) proposed that igneously differentiated asteroids and their surviving fragments were dynamically excited by planetary embryos at 1-2 AU and scattered into the asteroid belt where they were captured by planetary embryos in that region. To test this hypothesis with numerical simulations, these authors made many simplifying assumptions to track thousands of massless test bodies that interacted with a swarm of Moon- to Mars-sized planetary embryos for 10 Myr. They found that $10 \%$ of the test bodies originally at 1.5-2.0 $\mathrm{AU}$ were scattered into the main belt after $1 \mathrm{Myr}$. For the 1.0-1.5 AU zone, 1-2\% were injected into the main belt after $2 \mathrm{Myr}$, while for the 0.5-1.0 AU zone, 0.01-0.1\% reached the main belt after 6 Myr. More realistic simulations of Bottke et al. model have not been published. 
When the model of Bottke et al. (2006) for differentiated planetesimals was proposed, there was no meteorite evidence suggesting that differentiated asteroids had been disrupted within a few million years after they formed (although disruption at this time prior to emplacement in the asteroid belt was a key part of their model). However, evidence supporting the early disruption of iron meteorite parent bodies has since been obtained from cooling rates that were determined from $\mathrm{Fe}-\mathrm{Ni}$ phases and $\mathrm{U}-\mathrm{Pb}$ age dating. The cooling rates of group IVA irons are correlated with their bulk $\mathrm{Ni}$ and vary by a factor of $\sim 50$. This implies that these objects solidified and cooled with little or no silicate mantle (Yang et al. 2010a) possibly after a grazing collision with larger bodies that separated their core and mantle materials (Asphaug et al. 2006). In addition, the ${ }^{207} \mathrm{~Pb} /{ }^{206} \mathrm{~Pb}$ age of a troilite nodule in an IVA iron meteorite shows that these irons cooled within 2-3 Myr of the formation of Calcium-Aluminum-rich Inclusions (CAIs) (Blichert-Toft et al. 2010). A differentiated body that was small enough to have solidified and cooled in 2-3 Myr could not have retained sufficient heat released by ${ }^{26} \mathrm{Al}$ to have melted. Thus the antiquity of the IVA iron meteorite requires an early collision to separate core and mantle material. The evidence that the IVA parent body was disrupted within 2-3 Myr of CAI formation, when chondrites parent bodies were accreting, supports the idea that the iron meteorites are derived from bodies that did not form in the asteroid belt.

Another line of support for the model by Bottke et al. (2006) comes from the fact that iron meteorites have concentrations of moderately volatile siderophile elements that are lower than those in chondrites. For example, the most depleted groups, IVA and IV, have $\mathrm{Ge} / \mathrm{Ni}$ and $\mathrm{Ga} / \mathrm{Ni}$ ratios that are $10^{-2}$ to $10^{-4}$ times those in chondrites. These depletions are probably due to early accretion in a hot solar nebula, as the alternative explanation, the impact volatilization during mantle removal, fails to explain the large depletions in group IVB, which crystallized before mantle loss (Yang et al. 2010a). Thermal models for the solar nebula and calculated condensation temperatures for $\mathrm{Ga}$, Ge, and the other volatile siderophile elements suggest that the parent bodies of the IVA and IVB irons may have accreted 0.3 Myr after CAI formation at a distance of 0.9 AU from the Sun (Bland \& Ciesla 2010). Group IIAB and IIIAB irons have higher concentrations of Ga and Ge suggesting later accretion (0.5-1.0 Myr) after CAIs at 1.0 $\pm 0.1 \mathrm{AU}$. So, the chemical compositions of iron meteorites provide strong support for their origin in bodies that accreted closer to the Sun and earlier than the chondrites parent bodies.

Given these arguments favoring derivation of igneously differentiated asteroids and meteorites from the terrestrial planet region, it is important to test the Bottke et al. (2006) model in more detail as it neglected many processes that would have affected the growth of planetesimals and scattering of their fragments into the asteroid belt. Here we focus on the effects of the giant planets, which were completely ignored by Bottke et al. (2006). 
By not including these effects, this model implies that iron meteorites parent bodies have to be melted to form cores, solidified, broken open, and emplaced in the asteroid belt before the growing, or fully grown giant planets destabilize the asteroid belt, ending accretion there and remove protoplanetary objects. However, it is widely accepted that during the time that in the inner solar system, runaway growth among planetesimals was in progress to form planetary embryos (Safronov 1969; Greenberg et al. 1978; Wetherill \& Stewart 1989; Weidenschilling et al. 1997; Kokubo \& Ida 2002), giant planets were growing in the outer parts. As a result, the dynamics of planetary embryos, which are the facilitators of the outscattering and capture of planetesimals, could have been affected by these objects. This has been reported in the works of many authors including Wetherill (1996), Petit et al. (2001), Chambers \& Cassen (2002), Chambers (2003), Levison \& Agnor (2003), Raymond et al. (2004, 2009), O’Brien et al. (2006, 2007), and Morishima et al. (2008, 2010) who have shown that the gravitational perturbation of a fully formed Jupiter profoundly affects planetesimal scattering in the asteroid belt. A more complete model of the origin of the parent bodies of iron meteorites has to include the effects of growing giant planets as well. It would be important to examine whether as the giant planets grow, sufficient numbers of the parent bodies of iron meteorites will in fact be injected into the asteroid belt. This paper presents the results of the first attempt in modeling this process.

Our goal is to show the proof of concept. That is, we would like to show that a planet in an outer orbit will in fact affect the rate of the scattering of differentiated planetesimals into the inner asteroid belt. At the time that the model by Bottke et al was proposed, it was considered that the timescale for the formation of giant planets would be as long as in the original core-accretion scenario (i.e., 10 Myr, Pollack et al. 1996). As such, the iron meteorite parent bodies could form and be scattered into the asteroid belt before Jupiter would be large enough to deplete the mass of the entire belt. However, if the time of giant planet formation is short, as in the disk instability model (Boss 2000, 2001; Maver et al. 2002), or comparable with the average lifetime of disks around young stars (e.g., 3 Myr) 1 as in the models by Rice \& Armitage (2003), Alibert et al (2004), Hubickyj et al. (2005), Lissauer et al. (2009), and Movshovitz et al. (2010), the perturbing effect of giant planets during their growth may not be negligible. The goal of this paper is show the latter concept. As mentioned before, a realistic model requires the simulations to be carried out while giant planets are growing. This is a complicated task that requires complex computational programming and long time simulations. However, before taking up on such a project, which

\footnotetext{
${ }^{1}$ The observational estimates of the lifetimes of disks around young stars suggest a lifetime of 0.1-10 Myr, with 3 Myr being the age for which half of stars show evidence of disks (Strom et al. 1993; Haisch et al. 2001; Chen \& Kamp 2004).
} 
is beyond the scope of this paper, it proves useful to determine if the effect is large enough to be worthy of the effort. It would also be helpful to determine a minimum mass for the giant planet for which its perturbation will be non-negligible. The latter will allow us to estimate a timescale for the formation of this planet that would be consistent with the time of the formation of the parent bodies of iron meteorites. For these reasons, in this paper, we consider the planet to have been fully formed, and carry out simulations for different values of its mass. Also, in order to be able to compare our results with those of Bottke et al. (2006), we run simulations for $10 \mathrm{Myr}$.

The outline of this paper is as follows. In Section 2, we present our model and the initial set up of the simulations. A detail of our numerical integrations and results is given in Section 3. Section 4 concludes this study by discussing the implications of the results to the origin of the parent bodies of iron meteorites, and their connections to the time of giant planet formation.

\section{The Model and Initial Set Up}

Since we are interested in portraying the effect of an outer planet on the out-scattering of planetesimals from the terrestrial planet region into the inner asteroid belt, we considered a heuristic model consisting of the Sun, a disk of protoplanetary embryos, and a fully formed planet. The disk included over one hundred Moon- to Mars-sized objects and more than 1200 planetesimals. We randomly distributed planetary embryos between $0.5 \mathrm{AU}$ and $4 \mathrm{AU}$ with mutual separations of 3-6 Hill radii (figure 1). The masses of these objects were chosen to increase with their semimajor axes $(a)$ and the number of their mutual Hill radii $(\Delta)$ as $a^{3 / 4} \Delta^{3 / 2}$. The total mass of the protoplanetary disk was approximately 4 Earth-masses, and its surface density, normalized to $8.2 \mathrm{~g} \mathrm{~cm}^{-2}$ at $1 \mathrm{AU}$, was considered to be proportional to $r^{-3 / 2}$ (Weidenschilling 1977; Hayashi 1981). We assigned a randomly chosen eccentricity between 0 and 0.05 to each protoplanet, and assumed that the initial orbital inclinations of these objects were 0.1 degrees.

Following Bottke et al. (2006), the planetesimals were considered to be massless particles. Since we are interested in the out-scattering of these objects from the region interior to $2 \mathrm{AU}$, we only focused on that region and randomly distributed planetesimals between 0.5 AU and 2 AU. Similar to the embryos, the eccentricities of these bodies were chosen randomly from the range of 0 to 0.05 , and their initial inclinations were taken to be 0.1 degrees.

The planet was considered to be in the current orbit of Jupiter. As explained before, 
we did not model the actual growth of the planet. Instead, we assumed that the planet was fully formed and carried out simulation for different values of its mass ranging from 0.1 to 300 Earth-masses.

Prior to describing the results, it is important to note that because in our simulations, planetesimals were treated as test particles, the mutual interactions between these objects were ignored. The embryos, on the other hand, were in full gravitational interaction with one another. These objects were allowed to collide and merge, and they could also accrete planetesimals (the latter would not change their masses). Since the focus of our study is on identifying the effect of the outer planet on the out-scattering of planetesimals, to avoid computational complexities, we assumed that collisions between embryos were perfectly inelastic and resulted in the accretion of the colliding bodies. During the simulations, the perturbing effects of growing embryos were taken into account. However, in order to better portray the out-scattering of planetesimals, the changes in embryos' radii are not shown in the figures (the symbols for these objects are all identical in size).

We would also like to emphasize that in a realistic model, in addition to the effects of the giant planets, other processes such as gas drag, dynamical friction, breakage and re-accretion of colliding objects, and the effects of the size and spatial distribution of planetesimals and planetary embryos must also be taken into account. However, such inclusive and detailed modeling of the evolution of asteroid belt is beyond the scope of our paper. Our goal is merely to demonstrate that the perturbation of a giant planet is an important effect which cannot be ignored. In that respect, as we explained above, our model is heuristic, and the results of our simulations are only comparable with similar models with no giant planets.

\section{Results of Numerical Simulations}

We numerically integrated the orbits of the planetesimals, protoplanets, and the outer planetary body using the hybrid integrator of the N-body integrations package Mercury (Chambers 1999). The timestep of integrations was set to 6 days, and following Bottke et al. (2006), simulations were carried out for $10 \mathrm{Myr}$.

Figures 2 and 3 show the results of simulations for planetary masses of 0.5 and 1 Earthmass at 2, 5, and $10 \mathrm{Myr}$. (Graphs of the inclination vs semimajor axis show the same features as the eccentricity plots.) A comparison between the state of the surviving planetesimals and planetary embryos for each planetary mass at similar times indicates that for small values of the mass of the planet, the perturbing effect of this object is practically negligible. In these systems, the dynamics of planetesimals and their scattering to outer regions is 
primarily governed by their interactions with the planetary embryos. As shown in figure 2 , the majority of the planetesimals that were scattered into the inner asteroid belt were originally from the region of 1.5-2 $\mathrm{AU}$ (29\% in the simulations of 0.5 Earth-mass and $28 \%$ in the simulations of 1 Earth-mass). This is an expected result that is also consistent with the results reported by Bottke et al. (2006). As explained later, planetesimals from the 1-1.5 AU region also contributed. However, the contribution of these objects was small and appeared late during the simulations.

As expected, when the perturbing planet has a larger mass, its effect becomes more apparent. Simulations have shown that systems with planetary mass of 10 to $\sim 50$ Earthmasses form a transitional regime in which the perturbing effect of the planet appears mainly at late times during the integration. Figures 4 and 5 show a sample of the results. A comparison between the results shown in the top and middle panels of figure 4 with those of figure 2 indicates that although during the first $5 \mathrm{Myr}$, the dynamics of planetary embryos in the two figures is slightly different, the overall dynamics of their planetesimals is closely similar. This is primarily due to the fact that the perturbing effect of the planet will require some time to reach the planetesimals interior to $2 \mathrm{AU}$. As the planet interacts with planetary embryos, it excites the orbits of these objects, in particular those in the region of 3-4 AU, causing their orbital eccentricities to rise to high values. For a given planetary orbit, this orbital excitation is enhanced by the mass of the planet. Simulations show that while at the end of the $10 \mathrm{Myr}$ integration, in a system with a 1 Earth-mass planet, the orbital eccentricities of embryos at the outer region of the disk did not exceed 0.16, in a system with a 50 Earth-mass planet, the planetesimals eccentricities rose up to $\sim 0.6$. As a result, more than $30 \%$ of the embryos between $2 \mathrm{AU}$ and $4 \mathrm{AU}$ in this system were ejected (i.e., their aphelion distances become larger than $100 \mathrm{AU}$ ). The interaction among planetary embryos causes their dynamical excitation to spread to those in closer orbits which in turn affects the dynamics of planetesimals in those regions. Our simulations show that for systems with a planetary mass in the transitional regime, this process may take close to $10 \mathrm{Myr}$ (the bottom panels of figure 4).

In systems where the planet was as massive as a gas giant, the results were quite dramatic. Figures 6 and 7 show the results for planetary masses of 100 and 300 Earthmasses. As shown in figure 6, planetary embryos at 3-4 AU are strongly disturbed by the perturbation of the planet. During the first $2 \mathrm{Myr}$ of integration, $50 \%$ of these embryos were scattered out of the system. The rapid progression of dynamical excitation to embryos in closer distances during this time caused $40 \%$ of all embryos to leave the system. This number rose up to $58 \%$ after $5 \mathrm{Myr}$ and $70 \%$ after $10 \mathrm{Myr}$. At this time, almost all embryos in the region of 3-4 AU had been ejected, and only $38 \%$ of the original embryos in the region between $2 \mathrm{AU}$ and $3 \mathrm{AU}$ survived. 
The dynamical excitation and ejection of embryos had profound effects on the dynamics and out-scattering of planetesimals. While similar to previous cases, the majority of the planetesimals that were scattered to the inner asteroid belt, originated in the region of 1.5$2 \mathrm{AU}$, the number of these planetesimals was drastically reduced. Unlike the simulations of figure 2 where $\sim 28 \%$ of the planetesimals from the region 1.5-2 AU reached the inner asteroid belt (this number was $21 \%$ for the transitional case, figure 4 ), in the simulations with a giant planet, this number dropped to $12 \%$. This decreasing trend is also observed in the out-scattered planetesimals from the 1-1.5 AU region. Unlike the simulations of figures 2 and 4 where in average close to $5 \%$ of these objects were scattered into the region outside 2.1 AU, the highly excited embryos in the system with a giant planet caused only slightly over $1 \%$ of the planetesimals from the region 1-1.5 AU to reach the inner asteroid belt. In none of our simulations, planetesimal from 0.5-1 AU reached distances larger than 1.6 AU.

\section{Implications of the Results}

As mentioned earlier, a very important implication of the model by Bottke et al. (2006) is that in this model the destruction of the parent bodies of iron meteorites occurs in the terrestrial planet region and not in the asteroid belt. The small bodies that are generated in this way will then be scattered into the inner asteroid belt through their interactions with planetary embryos. As shown by our simulations, depending on its mass, an outer planet may play an important role in this process. The perturbation of this object may directly affect the dynamics of planetary embryos which will subsequently affect the interactions of these objects with planetesimals and the efficiency of their back-scattering from the terrestrial planet region into the inner asteroid belt. Integrations point to 50 Earth-masses as the lower limit for which the perturbation of the outer planet can no longer be ignored. In systems with a smaller planetary mass, particularly those with planets smaller than 10 Earth-masses, the dynamics of planetary embryos is primarily driven by their interactions with one another. In these systems, the efficiency of the scattering of planetesimals from terrestrial region into the inner asteroid belt increases as planetary embryos collide and grow to larger sizes. As the latter is a slow process, the implantation of planetesimals in the inner asteroid belt in these systems may require a long time.

It is important to note that the time of the delivery of differentiated planetesimals to the inner asteroid belt has to be consistent with cosmochemical data. This time is subject to two limiting factors; 1) the time of the cooling of iron meteorites, and 2) the time that it takes for the outer planet to grow to the critical mass of 50 Earth-masses. At the time of

the publication of the paper by Bottke et al. (2006), experimental data seemed to indicate 
that iron meteorites cooled over $100 \mathrm{Myr}$ and therefore their parent bodies survived for that period of time (Chabot \& Kaak 2006). However, in the past few years, new cooling rate data and radiometric ages have shown that the destruction of iron meteorites parent bodies must have begun much earlier, perhaps as early as a few million years after the formation of CAIs (Blichert-Toft et al. 2010; Yang et al. 2010b; Scott et al 2011). This implies that the process of the delivery of planetesimals to the inner asteroid belt must occur during the first few million years after the formation of CAIs, and before the outer planet reaches 50 Earth-masses. To constrain this time, we note that a comparison between the results of our simulations for all planetary masses show that no significant differences exist between the dynamical state of planetesimals during the first $2 \mathrm{Myr}$ (top panels of figures $2,4,6)$. In all our simulations, the gravitational effect of the perturbing planet becomes readily apparent after $5 \mathrm{Myr}$, and is far more pronounced after 10 Myrs. In other words, our simulations suggest that the period during which a significant number of planetesimals can be transferred from the terrestrial region into the inner asteroid belt has to be within the first 5 Myr. This is a time that is also consistent with the time of the destruction of the slow-cooling iron meteorites. Considering $\sim 50$ Earth-masses as the critical mass above which the perturbing effect of the planet cannot be ignored, the statement above suggests that in a more realistic model, where the effect of a growing planet in the orbit of Jupiter is considered, in order for the mechanism proposed by Bottke et al. (2006) to efficiently deliver planetesimals from the region interior to $2 \mathrm{AU}$ to the inner asteroid belt, the rate of the growth of the giant planet has to be such that the planet does not reach 50 Earthmass before the first $5 \mathrm{Myr}$. In other words, a giant planet formation scenario in which a planet in the orbit of Jupiter may grow to 50 Earth-masses in less than $\sim 5 \mathrm{Myr}$, may not be consistent with the time of the formation and cooling of the parent bodies of iron meteorites. It is important to caution that this result has been obtained using our simple model where effects of the circularization of the orbits of embryos due to their tidal interactions with the gaseous disk (Tanaka \& Ward 2004) and dynamical friction (Adachi et al. 1976) have been ignored. These effects, combined with the non-zero offset between the time-zero of the simulations and the true age of the system, may slightly change the above-mentioned 5 Myr timescale.

Our simulations also show that, regardless of the mass of the outer planet, the largest contribution to the scattered planetesimals from the terrestrial region into the inner asteroid belt $(<2.5 \mathrm{AU})$, came from the 1.5-2 AU zone. As mentioned in the previous section, the number of these objects decreases as the mass of the planet increases. This is primarily due to the fact that in systems with larger planetary mass, planetary embryos (the facilitators of the out-scattering of planetesimals) are dynamically excited and many of them are scattered out of the system. Simulations also indicate that in systems with small planets, where the 
effect of this body is negligible, a few planetesimals from the region of 1-1.5 AU reach the inner asteroid belt as well. Results show that the first of these objects entered the asteroid belt in approximately $5 \mathrm{Myr}$ after the start of the simulation. Given that the out-scattering of planetesimals is the result of their interactions with planetary embryos, it is expected that in systems where the outer planet is massive and planetary embryos are ejected from the system at the early stage of its dynamical evolution, it may take even loner for planetesimals from the region interior to $1.5 \mathrm{AU}$ to reach the inner asteroid belt (see the bottom panels of figure 6). In other words, planetesimals from the region of 1-1.5 AU could have contributed to the parent bodies of iron meteorites. But this contribution is very small. Any contribution from planetesimals in the region interior to $1 \mathrm{AU}$ is, however, highly unlikely.

In closing, we would like to mention that the effect of the migration of the giant planet, both during its growth and after its formation was not included in our simulations. While migration during formation, as suggested by Alibert et al (2004), may not be consistent with the model discussed here (these authors considered Jupiter to start its migration at $8 \mathrm{AU}$ with a mass equal to a fraction of Earth and to reside in its current orbit with its current mass after $1 \mathrm{Myr}$ ), post formation migration may have a profound effect on the outcome of our simulations. As suggested by Tsiganis et al. (2005) and Walsh et al (2011), giant planets in our solar system might have migrated after their formation (also see Morbidelli et al. 2005; Gomes et al. 2005). The inward migration of Jupiter in these cases, increases the perturbing effect of this object on the dynamics of planetary embryos (Gomes 1997), and extends the range of its perturbation to deeper regions interior to $2 \mathrm{AU}$. It is expected that in this case, the efficiency of the back-scattering of planetesimals is reduced even more drastically as obtained from our simulations.

We would like to thank the anonymous referee for critically reading our manuscript and for his/her constructive suggestions. We acknowledge support from NASA Cosmochemistry program under the grant NNH08ZDA001N-COS. NH also acknowledges support from NASA Astrobiology Institute (NAI) under Cooperative Agreement NNA04CC08A at the Institute for Astronomy (IfA), University of Hawaii (UH), and NASA EXOB grant NNX09AN05G.

\section{REFERENCES}

Adachi, I., Hayashi, C. \& Nakazawa, K. 1976, Prog. Theor. Phys., 56, 1756

Alibert, Y., Mordasini, C., \& Benz, W. 2004, A\&A, 417, L25

Asphaug, E., Agnor, C. B., \& Williams, Q. 2006, Nature, 439, 155 
Bland, P. A., \& Ciesla, F. J. 2010, Lunar Planet. Inst. Contribution No. 1533, 1817

Blichert-Toft, J., Moynier, F., Lee, C-T. A., Telouk, P., \& Albarde, F. 2010, E\&PSL, 296, 469

Boss, A. P. 2000, ApJL, 536, L101

Boss, A. P. 2001, ApJ, 563, 367

Bottke, W. F., Nesvorny, D., Grimm, R. E., Morbidelli, A., \& O’Brien, D. P., 2006, Nature, 439,821

Burbine, T. H., Meibom, A., \& Binzel, R. P. 1996, MAPS, 31, 607

Chabot, N. L., \& Haack, H. 2006, in: Meteorites and the Early Solar System II, D. S. Lauretta and H. Y. McSween Jr. (Eds.), Univ. Arizona Press, Tucson, pp.747

Chambers, J. E. 1999, MNRAS, 304, 793

Chambers, J. E., \& Cassen, P. 2002, MAPS, 37, 1523

Chambers, J. E. 2003, LIP contribution No. 2000

Chen, C. H., \& Kamp, I. 2004, ApJ, 602, 985

Gomes, R. S. 1997, AJ, 114, 396

Gomes, R. S., Levison, H. F., Tsiganis, K., \& Morbidelli, A. 2005, Nature, 435, 466

Greenberg, R., Hartmann, W. K., Chapman, C. R., Wacker, J. F. 1978, Icarus, 35, 1

Haisch Jr., K. E., Lada, E. A., \& Lada, C. J. 2001, ApJL, 553, L153

Hayashi, C. 1981, Prog. Theor. Phys. Suppl., 70, 35

Hevey, P. J., \& Sanders, I. S. 2006, MAPS, 41, 95

Hubickyj, O., Bodenheimer, P. \& Lissauer, J. J. 2005, Icarus, 179, 415

Kleine, T., Mezger, K., Palme, H., Scherer, E., \& Münker, C. 2005, Geochim. Cosmochim. Acta, 69, 5805

Kokubo, E., \& Ida, S. 2002, ApJ, 581, 666

Levison H. F., \& Agnor, C. 2003, AJ, 125, 2692 
Lissauer, J. J., Hubickyj, O., DÁngelo, G., Bodenheimer, P. 2009, Icarus, 199, 338

Markowski, A., Leya, I., Quitté, G., Ammon, K., Halliday, A. N., \& Wieler, R. 2006, E\&PSL,250, 104

Markowski, A., Quitté, G., Halliday, A. N., \& Kleine, T. 2006, E\&PSL, 242, 1

Mayer, L., Quinn, T., Wadsley, J., \& Stadel, J. 2002, Science, 298, 1756

Minton, D. A., \& Malhotra, R. 2009, Narure, 457, 1109

Morbidelli, A., Lavison, H. F., Tsiganis K., \& Gomes, R. S. 2005, Nature, 435, 462

Morishima, R., Schmidt, M. W., Stadel, J., \& Moore, B., 2008, ApJ, 685, 1247

Morishima, R., Stadel, J., \& Moore, B. 2010, Icarus, 207, 517

Moskovitz, N. A., Jedicke, R., Gaidos, E., Willman, M., Nesvorny, D., Fevig, R., \& Ivezić, Z. 2008, Icarus, 198, 77

Movshovitz, N., Bodenheimer, P., Podolak, M., \& Lissauer, J. J. 2010, Icarus, 209, 624

O’Brien, D. P., Morbidelli, A., \& Bottke, W. F. 2007, Icarus, 191, 434

O’Brien, D. P., Morbidelli, A., \& Levison, H. F. 2006, Icarus, 184, 39

Petit, J., Morbidelli, A., \& Chambers, J. E. 2001, Icarus, 153, 338

Pollack, J. B., Hubickyj, O., Bodenheimer, P., Lissauer J. J., Podolak, M., \& Greenzweig, Y. 1996, Icarus, 124, 62

Raymond, S. N., Quinn, T., \& Lunine, J. I. 2004, Icarus, 168, 1

Raymond, S. N., O’Brien, D. P., Morbidelli, A. \& Kaib, N. A. 2009, Icarus, 203, 644

Rice, W. K. M. \& Armitage, P. J. 2003, ApJ, 598, L55

Safronov, V. S. 1969, Evolution of Protoplanetary Cloud and Formation of the Earth and Planets, Nauka, Moscow

Scott, E. R. D. 2008, MAPS Supp., 43, paper id. 5284.

Scott, E. R. D., Goldstein, J. I. \& Yang, J. 2011, Lunar Planet. Inst. Contribution No. 1608, p. 2498 
Strom, S. E., Edwards, S., \& Skrutskie, M. F. 1993, In: E. H. Levy and J. I. Lunine (Eds) Protostars and Planets III, Univ. of Arizona Press, Tucson, pp. 837-866

Tanaka, H. \& Ward, W. R. 2004, ApJ, 602, 388

Tsiganis, K., Gomes, R. S., Morbidelli, A., \& Lavison, H. F. 2005, Nature, 435, 459

Walsh, K. J., Morbidelli, A., Raymond, S. N., O’Brien, D. P., \& Mandell, A. M., 2011, Nature, 475, 206

Weidenschilling, S. J. 1977, Astrophys. Space Sci., 51, 153

Weidesnchilling, S. J., Spaute, D., Davis, D. R., Marzari, F., \& Ohtsuki, K. 1997, Icarus, 128,429

Wetherill, G. W., \& Stewart, G. R. 1989, Icarus, 107, 117

Wetherill, G. W. 1996, Icarus, 119, 219

Yang, J., Goldstein, J. I., Michael, J. R., Kotula, P. G. \& Scott, E. R. D. 2010a, Geochim. Cosmochim. Acta, 74, 4493

Yang, J., Goldstein, J. I., \& Scott, E. R. D. 2010b, Geochim. Cosmochim. Acta, 74, 4471 


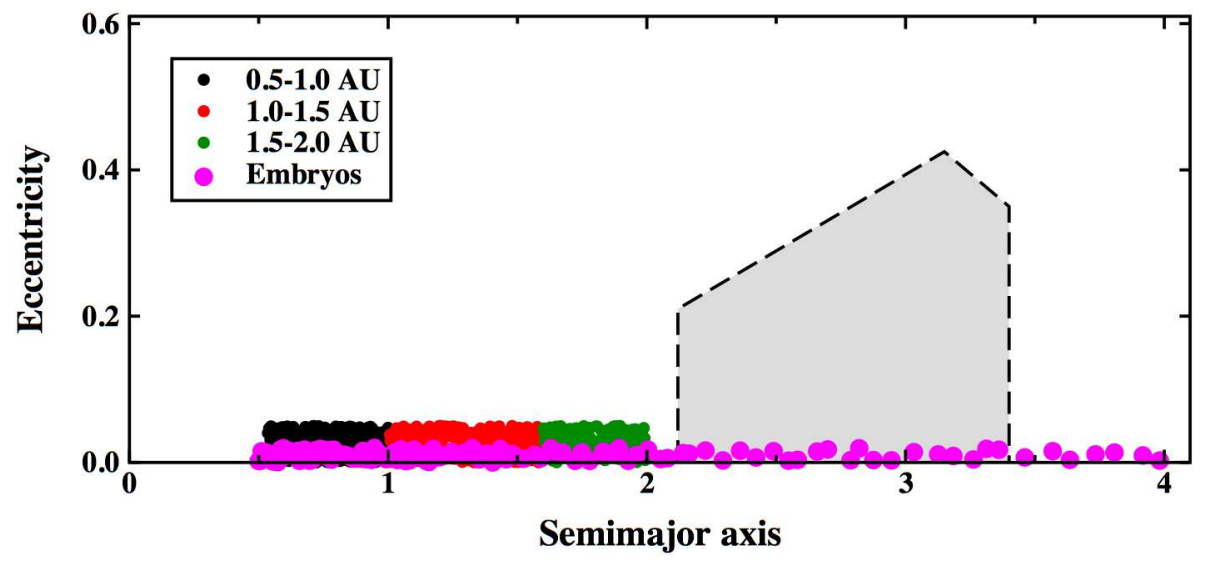

Fig. 1. - Initial distribution of planetesimals (black, red, green) and planetary embryos (pink). Note that because we are interested in the out-scattering of planetesimals from the terrestrial region into the asteroid belt, we distributed these objects only between $0.5 \mathrm{AU}$ and 2 AU. The gray area shows the asteroid belt. 

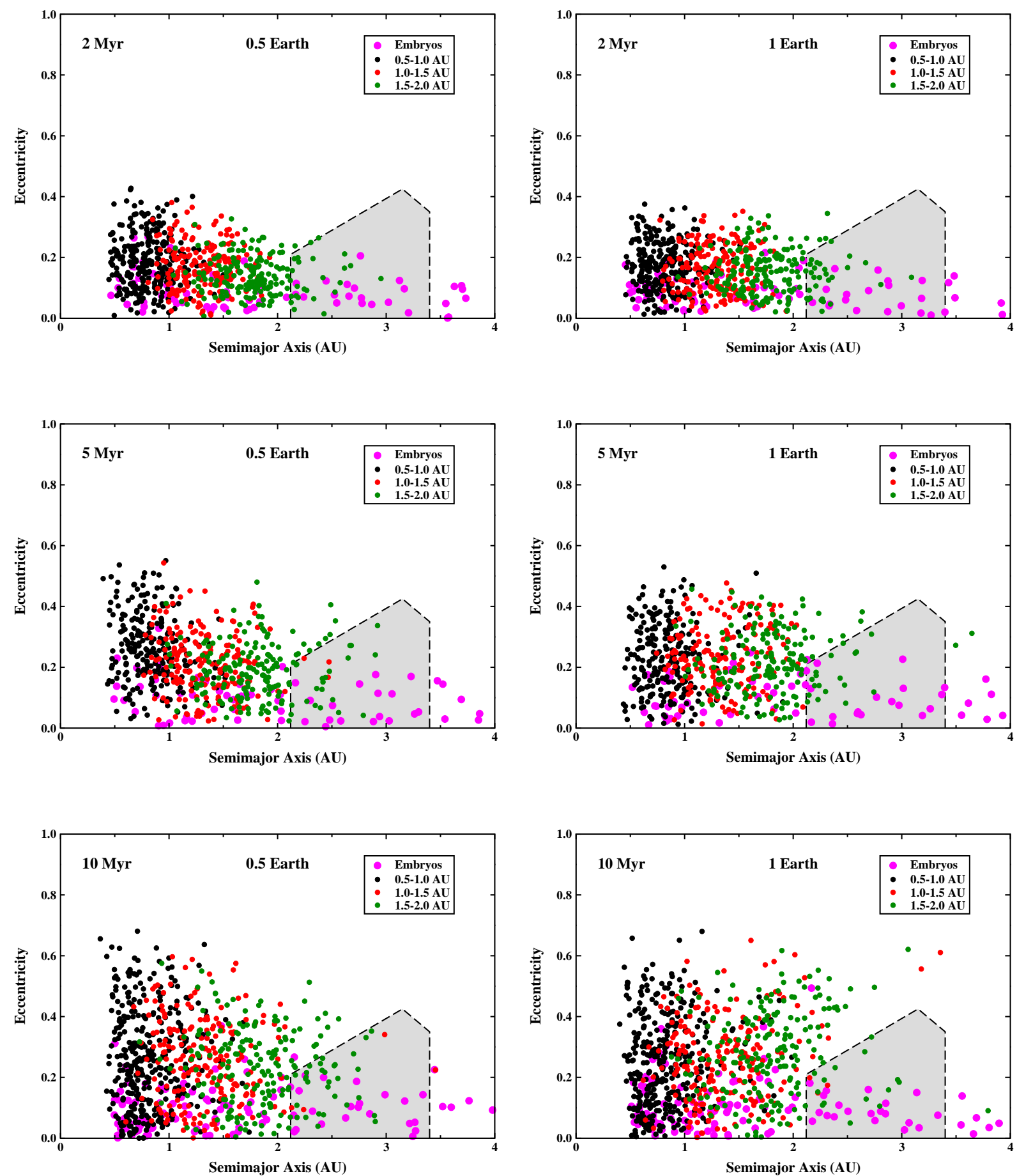

Fig. 2.- Snapshots of the dynamical state of planetesimals and planetary embryos in a system with a 0.5 Earth-mass (left) and a 1 Earth-mass (right) planet in the orbit of Jupiter. 

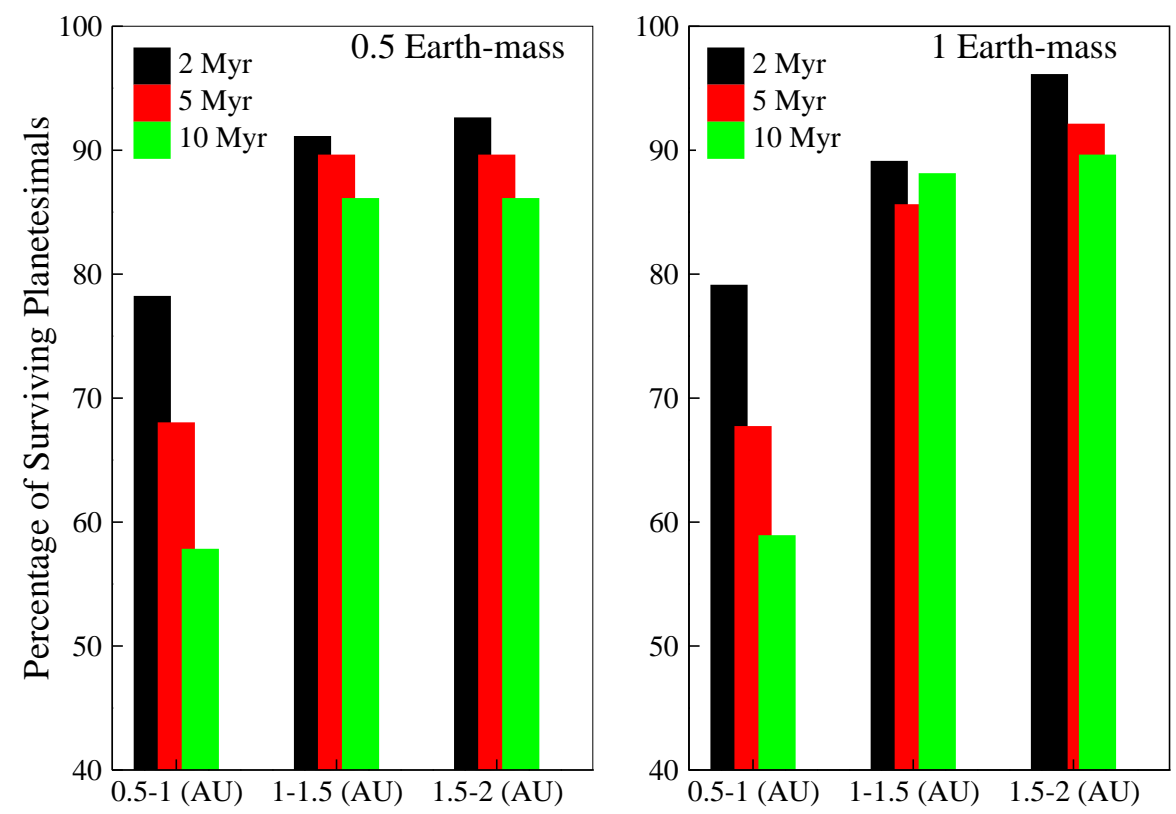

Fig. 3.- Percentage of survived planetesimals at the three zones 0.5-1, 1-1.5, and 1.5-2 (AU) for a perturber with a mass of 0.5 and 1 Earth-mass. 

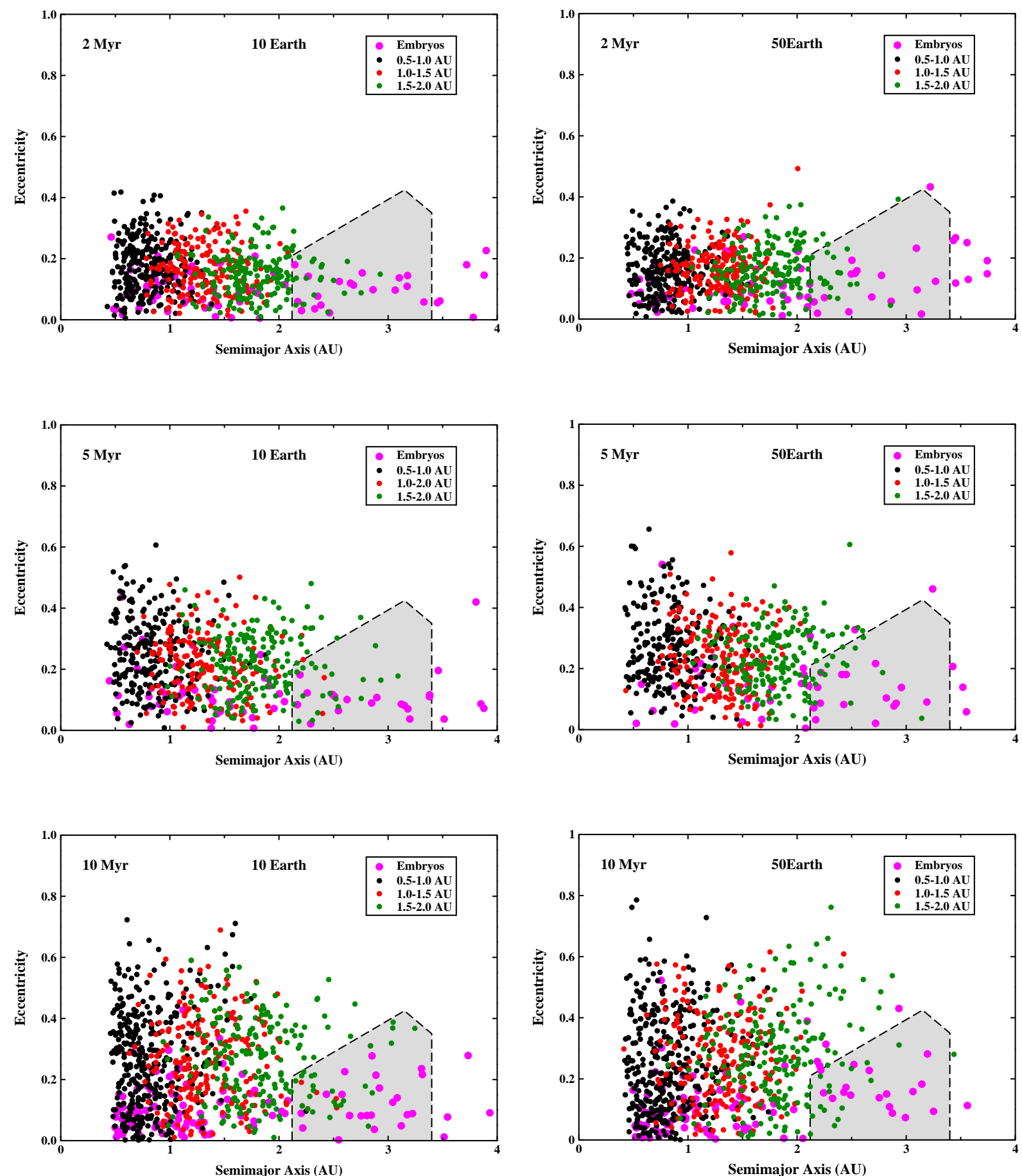

Fig. 4. - Same as figure 2. The planet has a mass of 100 Earth-masses on the left and 300 Earth-masses on the right. 

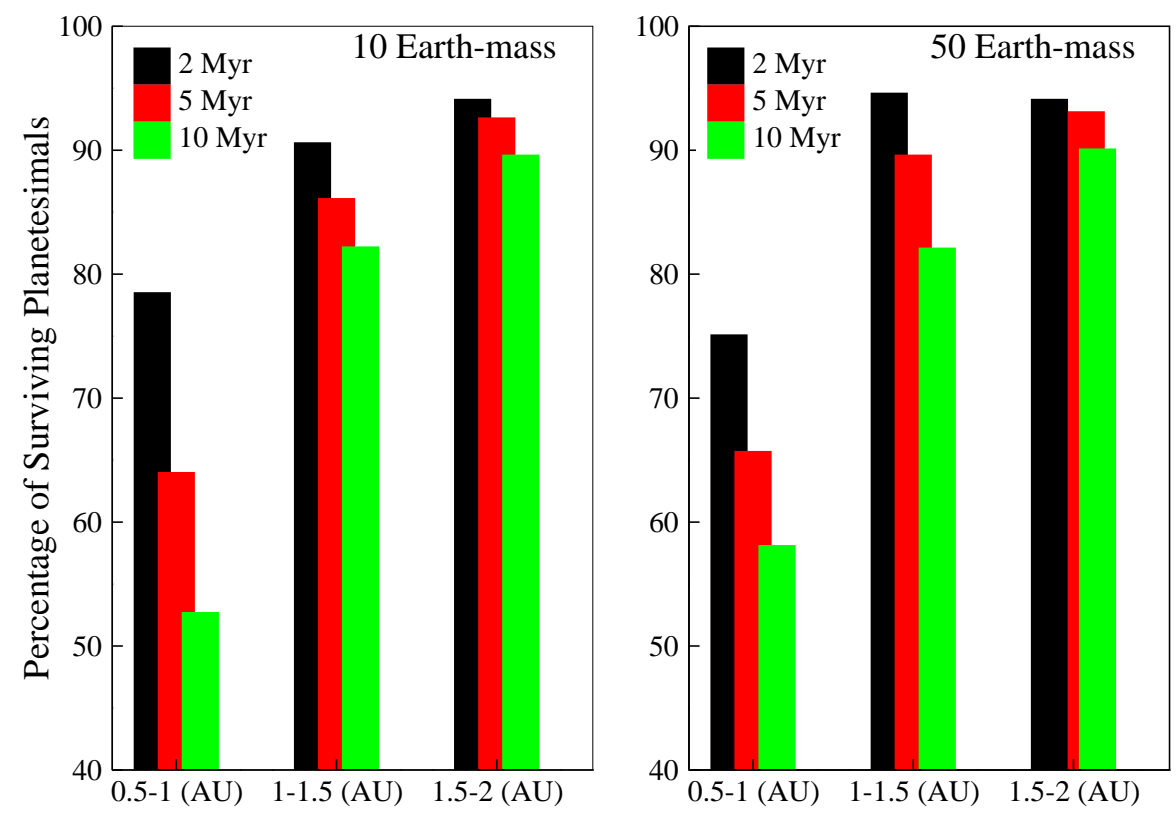

Fig. 5.- Percentage of survived planetesimals at the three zones 0.5-1, 1-1.5, and 1.5-2 (AU) for a perturber with a mass of 10 and 50 Earth-masses. 

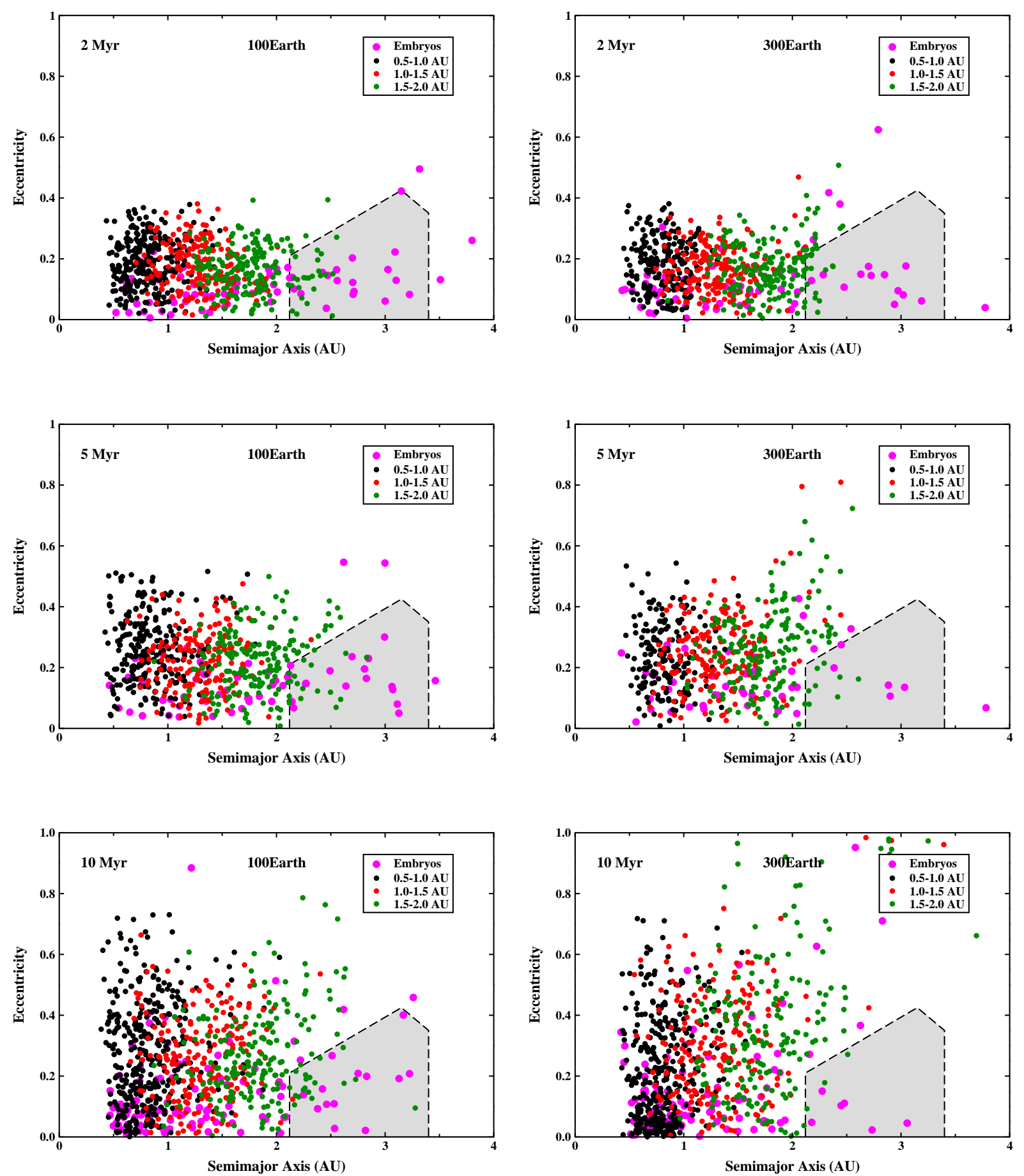

Fig. 6. - Same as figure 2. The planet has a mass of 100 Earth-masses on the left and 300 Earth-masses on the right. 

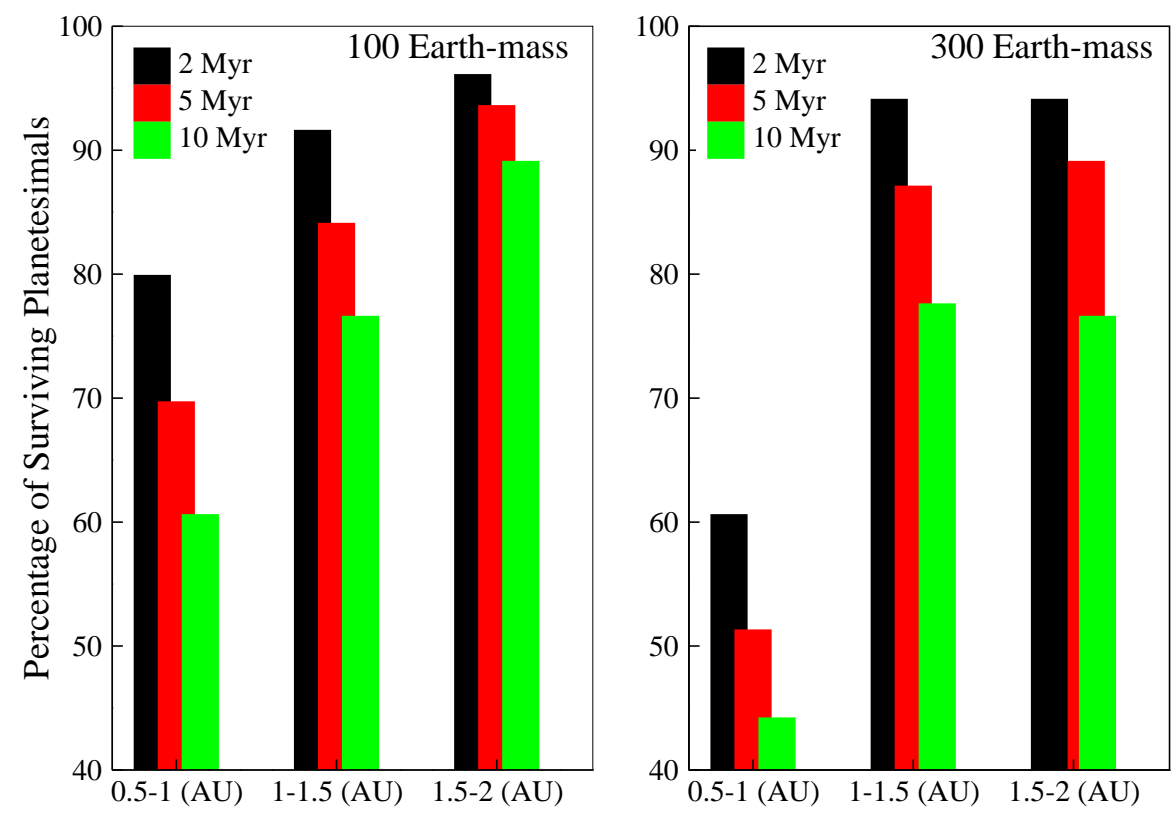

Fig. 7.- Percentage of survived planetesimals at the three zones 0.5-1, 1-1.5, and 1.5-2 (AU) for a perturber with a mass of 100 and 300 Earth-masses. 